\title{
Evaluation of Aberration-corrected Optical Sectioning for Exploring the Core Structure of $1 / 2[111]$ Screw Dislocations in BCC Metals
}

\author{
D. Hernandez-Maldonado ${ }^{1,2,}$; R. Gröger ${ }^{3}$; Q. M. Ramasse' ${ }^{1}$; P. B. Hirsch²; P.D. Nellist ${ }^{2}$ \\ 1. SuperSTEM Laboratory, STFC Daresbury Campus, Keckwick Lane, Daresbury, UK \\ 2. Department of Materials, University of Oxford, Parks Road, Oxford, UK \\ ${ }^{3 .}$ Institute of Physics of Materials and CEITEC, Academy of Sciences of the Czech Republic, Brno, \\ Czech Republic.
}

The introduction of spherical-aberration correctors in the Scanning Transmission Electron Microscope (STEM) has allowed an improvement in spatial resolution to the sub-angströn scale accompanied by a reduction of the depth of focus (due to the increase in probe convergence angles), which in a modern instrument is just a few nanometers, thus often less than the sample thickness. This can be exploited to extract information along the beam direction by focusing the electron probe at specific depths within the sample. Optical sectioning has been proved to be a powerful tool for the study of the core structure of screw dislocations. It has been used to observe the depth-dependence of the strain field due to the Eshelby twist associated with dislocations containing a screw component in thin STEM samples. The measurement of the magnitude of the displacement confirmed the screw Burgers vector for dislocations in $\mathrm{GaN}$ [1] and allowed the identification of a new dissociation reaction of mixed [c+a] dislocations [2]. The optical sectioning approach has also been applied to the direct observation of the c-component of the dissociation reaction of mixed $[\mathrm{c}+\mathrm{a}]$ dislocations in $\mathrm{GaN}$ by imaging a dislocation lying transverse to the electron beam [3].

In this communication we will evaluate the optical sectioning technique to explore the core structure of $1 / 2$ [111] screw dislocations in body-centred cubic (BCC) metals. The study of this structure is of high interest because the low-temperature plastic deformation of BCC metals is controlled by the glide of $1 / 2$ [111] screw dislocations. Their low mobility is caused by the non-planar nature of their cores [4] which are extended into several planes in the zone of the Burgers vector. The non-planar spreading of the core is characterized by displacements of the atoms around the core in the directions parallel and perpendicular to the dislocation line, also called screw and edge components respectively. In the last 47 years [5] these studies have been made by molecular statics using a number of different interatomic potentials. However, attempts at experimental observations by TEM have been hindered by the Eshelby twist effect [6,7]. The aim of this work is to investigate whether the edge and screw displacements associated with $1 / 2[111]$ screw dislocations in BCC metals can be detected by optical sectioning in highangle annular dark field (HAADF) imaging in STEM conditions.

Here we show, by using atomistic and image simulations, how this technique can be used to image the core structure of these screw dislocations at atomic resolution along a direction transverse to the screw axis. Figure 1 shows that the helicoidal displacements around the core of a $1 / 2$ [111] screw dislocation in Mo can be imaged with the dislocation lying transverse to the electron beam by optically sectioning the plane containing the dislocation. In order to reveal the effect of the spreading of the core, we have considered the atomic positions obtained from two different models. In the first, we treat the dislocation as a singularity in an anisotropic elastic continuum for which the displacement field around the dislocation is well-known [8]. The second model utilizes the Bond Order Potential for Mo [9] to determine the relaxed core of a non-singular dislocation. This is shown to be spread on three $\{110\}$ planes in the zone of the [111] axis. The edge and screw displacements generated by the core relaxation 
are obtained by subtracting the atomic positions obtained in the second model from those in the first model. Both edge and screw displacements are plotted in Figure 2, revealing that the non-planar character of the core has a bigger impact on the edge component, reaching a maximum displacement of $11 \mathrm{pm}$ due to core relaxation. The experimental conditions under which these displacements could be directly detected using aberration-corrected STEM will be discussed [10].

\section{References:}

[1] J. G. Lozano, et al., Phys. Rev. Lett. 113 (2014) 135503.

[2] P.B. Hirsch, et al., Philos. Mag. 93 (2013) 3925.

[3] H. Yang, et al., Nature Commun. 6 (2015) 7266.

[4] P.B. Hirsch, Fifth Internat. Congs. Crystallography, Cambridge, 139 (1960)

[5] Vitek, V. et al., Philos. Mag. 21, (1970) 1049.

[6] Sigle, W., Philos. Mag. 79, (1999) 1009.

[7] Mendis, B. G et al., Philos. Mag. 86, (2006) 4607.

[8] Stroh, A. N., J. Math. Phys. 41 (1962) 77.

[9] Lin, Y.-S., Mrovec, M., Vitek, V., Model. Simul. Mater. Sci. Eng. 22, (2014) 034002.

[10] The SuperSTEM Laboratory is the U.K National Facility for Aberration-Corrected STEM, supported by the Engineering and Physical Science Research Council (EPSRC).

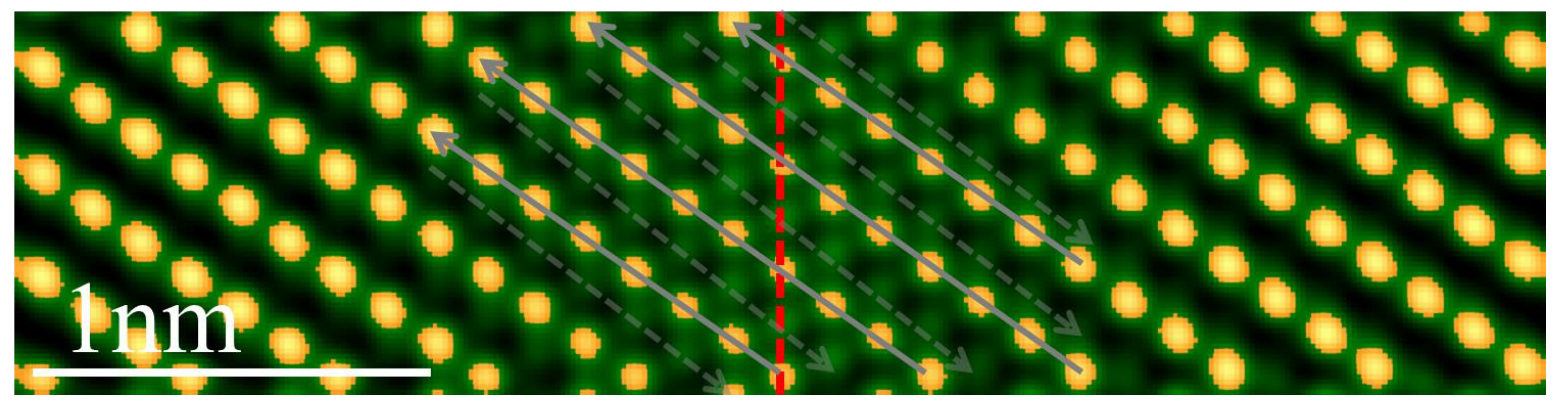

Figure 1. Simulated false coloured HAADF image along [-101] using a model of Mo with a 1/2[111] screw dislocation (red dashed line) placed in the middle of the foil along the direction normal to the plane of the figure. The thickness of the block is $10 \mathrm{~nm}$ along the direction parallel to the electron beam. The defocus value is $5 \mathrm{~nm}$. Due to the finite depth of focus it is possible to observe displacements on both sides of the dislocation. One side of the crystal (in which the focal-plane lies) is displayed in

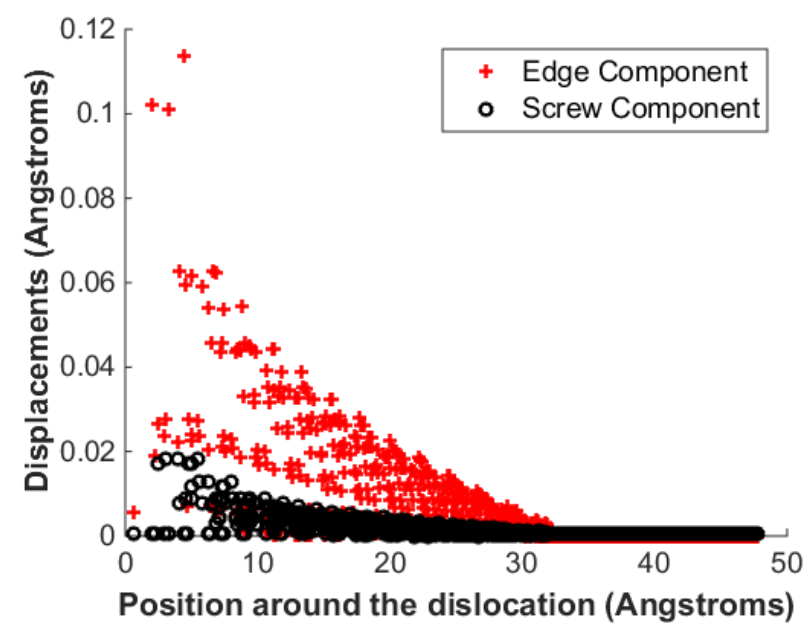
orange; the other side in green. Grey arrows are added to visualize the helicoidal pattern characteristic of screw dislocations.

Figure 2. Screw and edge components of the displacement field around the screw dislocation caused by molecular statics relaxation (in absolute values). The horizontal axis represents the radial distance of each atom from the centre of the dislocation that is placed at the origin. The vertical axis represents the displacements of atoms in the relaxed block relative to the anisotropic linear-elastic solution. 